\title{
Minimally invasive implantation of a cardioverter-defibrillator in a small patient
}

Christopher S. Snyder, MD, ${ }^{\text {a }}$ Victor Lucas, MD, ${ }^{a}$ Thomas Young, MD, ${ }^{a}$ Rani Darling, RN, ${ }^{a}$ Geeta Dalal, MDa, and

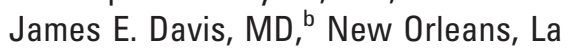

$\mathrm{T}$ he patient is a 3-year-old girl weighing $13 \mathrm{~kg}$, with a diagnosis of long QT syndrome, who experienced an episode of syncope and was found to be in ventricular fibrillation. She was cardioverted and transported to our institution. On arrival, she was taken to the operating room for implantation of an epicardial cardioverter-defibrillator. Because of her small size, this implantation was performed by using a previously unreported minimally invasive technique.

\section{Clinical Summary}

In the operating room general anesthesia was achieved. A midline incision was made over the xiphoid that extended $3 \mathrm{~cm}$ inferiorly. The linea alba was divided, and the retrosternal space was entered and developed. A 3-cm longitudinal pericardial incision was made, and a retractor was placed inside the pericardial sac to elevate the sternum.

A 58-cm dual-coil shocking lead (Medtronic 6949) was looped around the ventricles, with the distal coil coming to rest on the anterior right ventricular wall and the more proximal portion of the lead emerging from the posterior pericardial sac along the diaphragm. The distal coil was secured with an epicardial-encircling suture. The midportion of the lead was fixed to the diaphragmatic surface of the right ventricle. The lead was then looped along the right ventricular diaphragmatic surface, positioning the proximal coil from the apex to the base of the heart. Additional epicardial sutures were used to secure the coil along the inferior ventricular surface. This maneuver allowed sufficient myocardial mass to be interposed between the coils.

A 35-cm bipolar, steroid-eluting pace-sense lead (Medtronic 4968) was sutured to the anterior right ventricular wall. On testing, the lead had excellent sensing and pacing thresholds. The implantable cardioverter-defibrillator (ICD) pocket was made in the left abdominal wall. The leads were tunneled from the preperitoneal space into the pocket. The pacing portion of the defibrillation lead was capped, and the coil leads were inserted into the pulse generator (En-Trust, Medtronic). The

From the Divisions of Pediatric Cardiology ${ }^{\mathrm{a}}$ and Cardiovascular Surgery, ${ }^{\mathrm{b}}$ the Ochsner Clinic Foundation, New Orleans, La.

Received for publication Dec 21, 2006; accepted for publication Jan 2, 2007.

Address for reprints: Christopher Snyder, MD, Ochsner Clinic Foundation, Department of Pediatric Cardiology, 1514 Jefferson Highway, New Orleans, LA 70121 (E-mail: csnyder@ochsner.org).

J Thorac Cardiovasc Surg 2007;133:1375-6

$0022-5223 / \$ 32.00$

Copyright () 2007 by The American Association for Thoracic Surgery doi:10.1016/j.jtcvs.2007.01.042

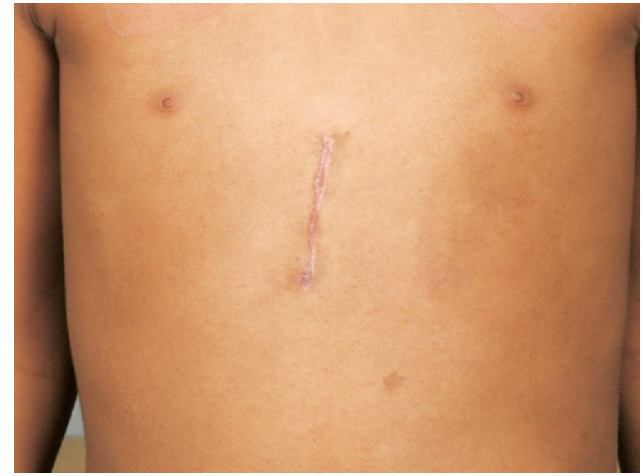

Figure 1. Residual scar post ICD implant. ICD, Implantable cardioverter defibrillator.

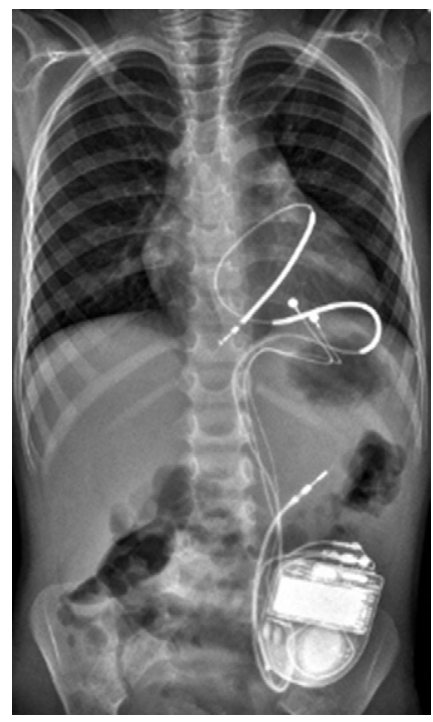

Figure 2. X-ray illustrating ICD and lead placement. ICD, Implantable cardioverter defibrillator.

bipolar ventricular pace-sense lead was inserted into the pacing position (Figure 1).

At this point, the ICD was tested 2 times. Ventricular fibrillation was induced, and the device detected all beats of the tachycardia and delivering a 15 -joule shock, successfully restoring sinus rhythm both times. After fascia and skin closure, the patient was extubated in the operating room (Figure 2). 


\section{Discussion}

In recent years, the number of patients meeting the criteria for implantation of an ICD has increased dramatically. A number of creative implantation methods have been reported, but each is faced with its own set of potential complications. ${ }^{1-3}$ These complications range from postpericardiotomy syndrome ${ }^{4}$ to lead or patch trauma and failure. ${ }^{5}$

There are a number of advantages to this implantation, the first of which is that the patient avoids a median sternotomy, and the second of which is that no subcutaneous array is required, therefore eliminating the possibility of array failure.

This case is evidence that a minimally invasive approach to pediatric ICD implantation can be successful. In a patient determined to be too small for traditional transvenous implantation or with a lack of venous access, this method of ICD implantation should be considered.

\section{References}

1. Cannon BC, Friedman RA, Fenrich AL, et al. Innovative techniques for placement of implantable cardioverter-defibrillator leads in patients with limited venous access to the heart. Pacing Clin Electrophysiol. 2006;29:181-7.

2. Berul CI, Triedman JK, Forbess J, et al. Minimally invasive cardioverter defibrillator implantation for children: An animal model and pediatric case report. Pacing Clin Electrophysiol. 2004;24:1789-94.

3. Luedemann M, Hund K, Stertmann W, et al. Implantable cardioverter defibrillator in a child using a single subcutaneous array lead and an abdominal active can. Pacing Clin Electrophysiol. 2004;27:117-9.

4. Stefanelli CB, Bradley DJ, Leroy S, et al. Implantable cardioverter defibrillator therapy of life-threatening arrhythmias in young patients. J Interv Card Electrophysiol. 2002;6:235-44.

5. Kettering K, Mewis C, Dornberger V, et al. Long-term experience with subcutaneous ICD leads: a comparison among three different types of subcutaneous leads. Pacing Clin Electrophysiol. 2004;27: $1355-61$.

\title{
Argatroban anticoagulation for renal replacement therapy in patients with heparin-induced thrombocytopenia after cardiovascular surgery
}

\author{
Andreas Koster, MD, PhD, ${ }^{a}$ Thomas Hentschel, MD, ${ }^{a}$ Tom Groman, MD, ${ }^{\mathrm{b}}$ Hermann Kuppe, MD, \\ Roland Hetzer, MD, PhD, ${ }^{b}$ Sebastian Harder, MD, PhD, ${ }^{\mathrm{c}}$ and Karl-Georg Fischer, MD, PhD, ${ }^{\mathrm{d}}$ \\ Berlin, Frankfurt/Main, and Freiburg, Germany
}

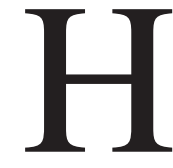
eparin-induced thrombocytopenia (HIT) is a severe thrombogenic disease requiring the use of alternative anticoagulants. Argatroban is a synthetic monovalent direct thrombin inhibitor indicated for prophylaxis and treatment of thrombosis in patients with HIT. Renal failure requiring renal replacement therapy (RRT) is a frequent complication in patients in whom HIT develops after cardiovascular surgery. The hepatic metabolism and relative short elimination half-life of 45 minutes render argatroban an interesting option for anticoagulation during RRT. However, there are scant data on dosing, pharmacokinetics, safety, and efficacy of argatroban in this indication. We report our initial experience with argatroban anti-

From the Departments of Anesthesia ${ }^{\mathrm{a}}$ and Cardiothoracic and Vascular Surgery, ${ }^{\mathrm{b}}$ Deutsches Herzzentrum Berlin, the Department of Clinical Pharmacology, Klinikum, J.W. Goethe-Universität Frankfurt/Main, ${ }^{\mathrm{c}}$ and the Department of Medicine, Division of Nephrology and General Medicine, Universitätsklinikum Freiburg, Germany. ${ }^{\mathrm{d}}$

The study was supported by the Deutsches Herzzentrum Berlin, Germany. Received for publication Nov 16, 2006; accepted for publication Jan 2, 2007.

Address for reprints: Andreas Koster, MD, PhD, Deutsches Herzzentrum Berlin, Augustenburger Platz 1, D-13353 Berlin, Germany (E-mail: koster@dhzb.de).

J Thorac Cardiovasc Surg 2007;133:1376-7

$0022-5223 / \$ 32.00$

Copyright $\odot 2007$ by The American Association for Thoracic Surgery doi:10.1016/j.jtcvs.2007.01.008 coagulation for continuous veno-venous hemofiltration (CVVH) and intermittent hemodialysis (IHD) in 10 patients diagnosed with HIT after cardiovascular surgery in whom acute renal failure developed necessitating RRT.

\section{Patients and Methods}

After approval by the local ethics committee and having obtained informed consent, we analyzed the data of 10 patients. In accordance with the study protocol, data were collected for a maximum of 30 days during RRT. Clinical follow-up of patient outcome was performed for 3 months after initiation of RRT with argatroban. All patients had thrombocytopenia after surgery or presented persistent thrombocytopenia postoperatively. In all patients the diagnosis of HIT was confirmed in an antibody assay (Particle Gel Immune Assay; DiaMed, Cressier sur Morat, Switzerland) and in the heparin-induced platelet aggregation assay. The decision to perform CVVH (Ultraflux AV 600S; polysulfone high flux hemodialyzer, Fresenius Medical Care AG, Bad Homburg, Germany) or IHD (Polyflux $140 \mathrm{H}$; polyamix high flux hemodialyzer, Gambro, Hechingen, Germany) was based on stability of hemodynamics and mobility of the patient. In general, RRT was started with CVVH and changed to IHD after hemodynamics were stable and patient mobility increased. Only in 1 patient was RRT initiated with IHD. Target activated partial thromboplastin time (aPTT) (Roche Diagnostics, Mannheim, Germany, normal value 20-22 seconds) during RRT was 50 to 80 seconds and 40 to 60 seconds in periods without CVVD or IHD. Before the start of RRT, argatroban administration was started with a continuous infusion of $1 \mu \mathrm{g} \cdot \mathrm{kg}^{-1} \cdot \mathrm{min}^{-1}$ for 1 hour. Thereafter, the aPTT was 